\title{
Electron temperature measurements in a copper chloride laser utilizing a microwave radiometer*
}

\author{
Emilio Sovero, Che Jen Chen, and F. E. C. Culick \\ California Institute of Technology, Pasadena, California \\ (Received 17 May 1976)
}

\begin{abstract}
The electron temperature as a function of laser tube temperature of a copper vapor laser utilizing copper chloride as a lasant has been obtained by measuring the microwave incoherent radiation from the plasma in the laser tube. An unexpected increase of electron temperature at higher laser tube temperature may be due mainly to Penning ionization of the $\mathrm{Cu}$ atom by the metastable state of $\mathrm{He}$ or $\mathrm{Ne}$ buffer gases. The results obtained in this work provide part of the basis for understanding the behavior of the laser output as a function of tube temperature.
\end{abstract}

PACS numbers: $42.60 . \mathrm{Cz}, 52.70 . \mathrm{Gw}, 32.10 . \mathrm{Lt}, 32.10 . \mathrm{Rz}$

\section{INTRODUCTION}

The previous experimental results ${ }^{1}$ for a copper vapor laser using copper chloride as the source of copper atoms indicated that there is an optimum tube temperature of about $400^{\circ} \mathrm{C}$ at which the laser power is maximum. It is understandable that at low temperature the copper chloride vapor pressure is too low to provide enough copper atoms for lasing; however, the reason for the power decrease above $400^{\circ} \mathrm{C}$ is not clear. It has been mentioned in Ref. 1 that a possible reason could be changes in the electron temperature when the tube temperature is varied. This experiment is designed to measure the electron temperature and laser output at different tube temperatures, to clarify this problem.

\section{THEORY OF MEASUREMENT}

A technique using the incoherent radiation in the region of microwave frequencies emitted from a plasma to measure the electron temperature in the plasma has been investigated by previous authors. ${ }^{2,3}$ The origin of the incoherent radiation in the plasma in the absence of an external electric or magnetic field is primarily the free-free collisions of electrons in the fields of ions and atoms. Therefore, the plasma radiation energy, which is a function of the radiation temperature of the radiators, is related to the mean electron energy. When the electrons have a Maxwellian distribution of velocities the radiation temperature is equal to electron temperature. The detailed description of the theory and validity of the measurement can be found in Refs. 2-4. A brief summary is presented here.

In the Rayleigh-Jeans limit $(h \nu \ll k T)$, which is generally appropriate for microwave radiation, the blackbody radiation intensity per unit solid angle per unit radian frequency interval $v \rightarrow v+\Delta v$ reduces to

$$
B\left(w, T_{r}\right)=k T_{r} v^{2} / 2 c^{2} .
$$

The radiation intensity from a plasma, by Kirchhoff's law, can be expressed as

$$
P_{w}=B\left(w, T_{r}\right) A_{w},
$$

where $\nu$ is the frequency in cycles per second, $k$ is the Boltzmann constant, $c$ is the velocity of light, and $A_{w}$ is the absorptivity of the plasma. The radiation tem- perature $T_{r}$ is defined $a s^{5}$

$$
k T_{r}=m \int_{0}^{\infty} Q(v) f v^{5} d v \quad\left(\int Q(v) \frac{\partial f}{\partial v} v^{4} d v\right)^{-1} .
$$

Here, $Q$ is the total collision cross section for momentum transfer for an electron with the other plasma constituents defined as follows: $m$ is the electronic mass; $v$ is the velocity of the electron; and $f$ is the electron distribution function, normalized so that $\int_{0}^{\infty} 4 \pi f v^{2}$ $d v=1$. It can be shown from Eq. (3) that (i) $T_{r}=T_{e}$ (electron temperature) if $f$ is Maxwellian and (ii) $\frac{3}{2} k T_{r}$ is the average electron energy if $Q \sim 1 / v$, regardless of the electron distribution function, Under the present experimental condition the electron energy distribution might not be Maxwellian. However, the measured radiation temperature is equal to the mean energy of electrons in He which has a $Q$ dependence of $1 / v$ and is approximately equal to the mean energy in $\mathrm{Ne}$ (see discussion in Ref. 2).

\section{EXPERIMENT}

\section{A. Description of the instrumentation}

A schematic diagram of the experimental setup is shown in Fig, 1. The laser tube $(25 \mathrm{~cm}$ long, $1 \mathrm{~cm}$ in diameter) is enclosed in a microwave cavity which has a tottal enclosure area of about $60 \mathrm{~cm}^{2}$. The area of the outlet of the waveguide is about $0.46 \mathrm{~cm}^{2}$. It is assumed that the microwave radiation in the cavity is in equilib-

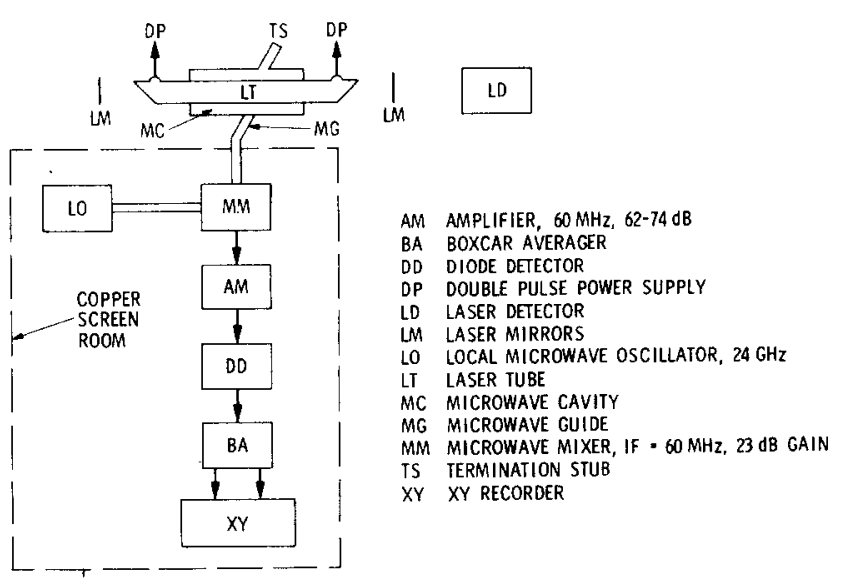

FIG. 1. Schematic diagram of experimental setup. 


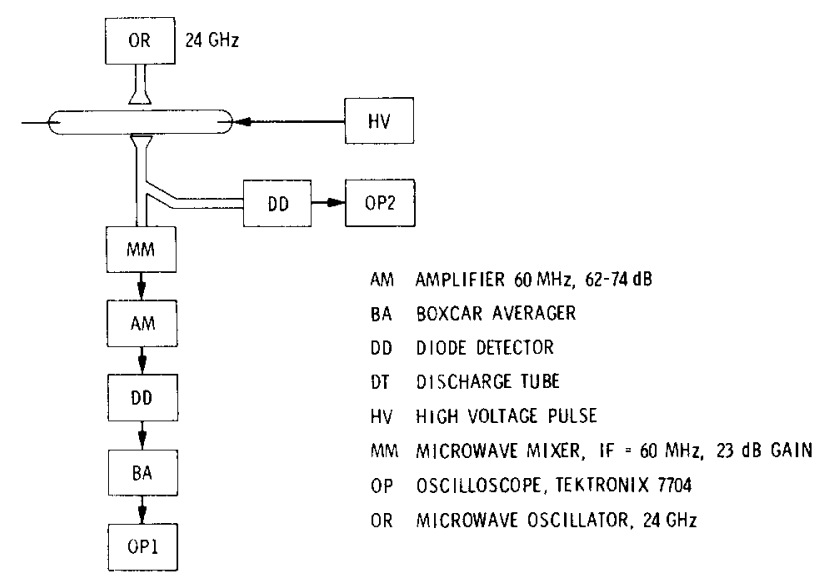

FIG. 2. Schematic diagram of the instrumentation for timedelay measurement.

rium with the plasma radiation and that the measured radiation leaking into the wave guide is a true sample of radiation in the plasma tube. The description of the laser tube operation has been published elsewhere ${ }^{1}$ and is omitted here. The frequency used in the microwave local oscillator is $24 \mathrm{GHz}$. The heterodyne intermediate frequency is $60 \mathrm{MHz}$. The built-in if amplifier in the microwave mixer has a gain of $23 \mathrm{~dB}$. A boxcar averager (made by Princeton Applied Research Co.) is used to improve the signal-to-noise ratio of the measurement. The averager samples synchronously the output signal from the diode with an aperture that can be fixed at any point on the output signal. The sampled signal is then applied to a variable time constant integrator, which puts out an average of a large number of repetitions of the signals over the aperture duration. The averaged signal has a higher signal-to-noise ratio because the average value of noise over a large number of repetitions is zero. The final radiation signal is displayed on a $X-Y$ recorder. The output signal from the superheterodyne radiometer is proportional to the input voltage, and thus to the square root of the radia tion temperature $T_{r}$. The laser detector (LD) is a fastrise-time (a fraction of a nanosecond) photodiode manufactured by Korad Co. All instrumentation is enclosed in a copper-screen room to minimize electric

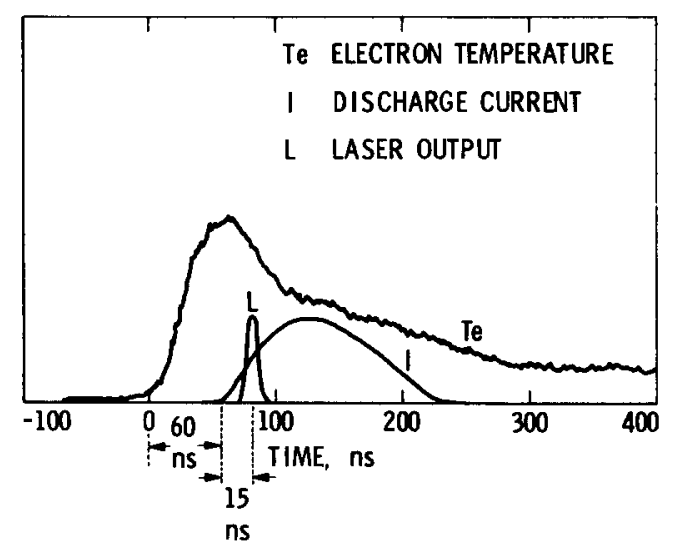

FIG. 3. Temporal relation among electron temperature, current peak, and laser output.

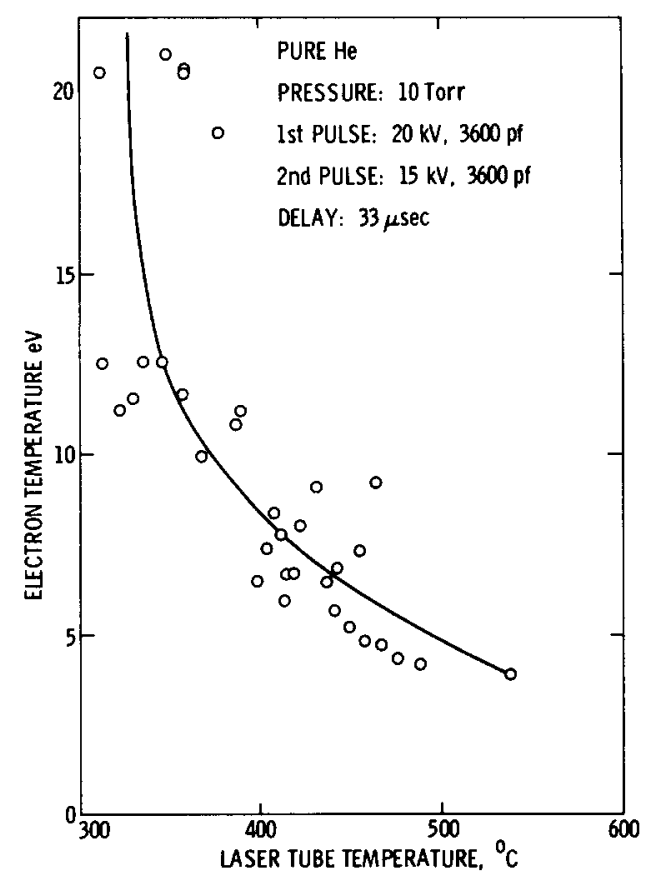

FIG. 4. Electron temperatures as function of laser tube temperature for pure He.

disturbance from the high-voltage discharge in the laser tube.

\section{B. Required calibration of the instruments}

\section{Correlation between the radiation temperature and strength of the output signal}

The laser discharge tube is operated in dc mode with pure helium gas; the discharge current and gas pressure are known. The radiation temperature under this condition is well established both by theoretical prediction $^{5}$ and experimental verification. ${ }^{5}$ The output signal is measured. Thus the strength of the output signal can be correlated with the radiation temperature under the same geometrical and electronic conditions. The calibration has also been checked with a neon standard noise source. The agreement between the two calibrations is satisfactory.

\section{Signal delay time in the electronic circuit}

Because the time scale in the present measurement and the signal delay time in the electronic circuit are of the same order, the correction of the time measurement for the signal delay time is required. The signal delay time in the circuit between the input of the microwave mixer to the output of boxcar averager is measured by using the scheme depicted in Fig. 2. The high-voltage pulses break down the discharge tube, causing the electron density in the tube to increase rapidly. The transmission of microwaves across the tube is cut off abruptly when the electron number density reaches the cutoff point. This cutoff point is observed simultaneously with oscilloscopes OP 1 and OP 2. The difference of the arrival time of the cutoff point in oscilloscopes OP 1 and OP 2 is measured. The signal delay time in the OS 2 is calculated from the length of 


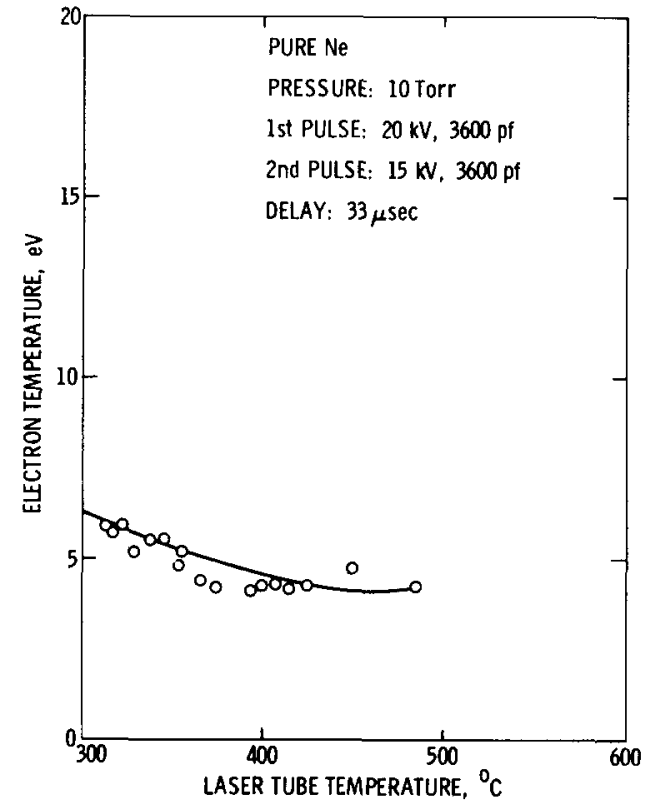

FIG. 5. Electron temperatures as function of laser tube temperature for pure $\mathrm{Ne}$.

coaxial cable used $(1.5 \mathrm{~ns} / \mathrm{ft})$. Thus the signal delay time in the electronic circuit is obtained.

\section{RESULTS AND DISCUSSIONS}

\section{A. Electron temperature dependence of the laser tube temperature}

The typical temporal relations among the peak values of the electron temperature, discharge current, and laser output are shown in Fig. 3. The maximum electron temperatures as a function of laser tube temperature for pure helium, neon and copper chloride are shown in Figs. 4-6, respectively. Despite the scatter

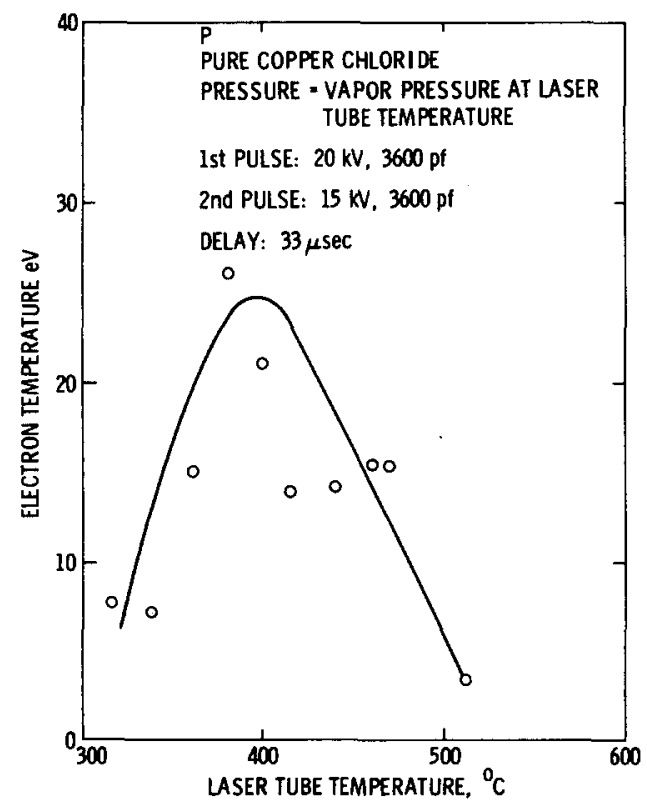

FIG. 6. Electron temperature as function of laser tube temperature for pure copper chloride.

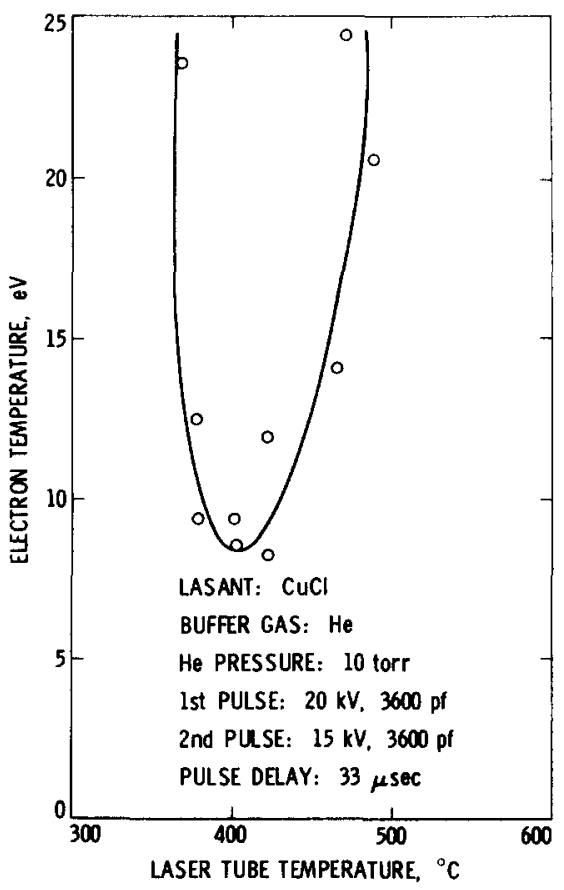

FIG. 7. Electron temperature as function of laser tube temperature for mixture of copper chloride and $\mathrm{He}$.

in the experimental data, the general trends agree with Von Engel's theory. ${ }^{6,7}$ According to Ref. 7, the electron temperature decreases as the laser tube temperature increases. A deviation from this trend at very low gas pressure has been observed previously ${ }^{2}$ and also in the present experiment with copper chloride as shown in Fig. 6; at lower temperatures, the electron temperature decreases as the laser tube temperature decreases.

The maximum electron temperature as a function of the laser tube temperature for mixtures of copper chloride and buffer gases $\mathrm{He}$ or $\mathrm{Ne}$ is shown in Figs. 7 and 8 , respectively. It can be seen that at the lower laser tube temperatures the descending portion of the elec-

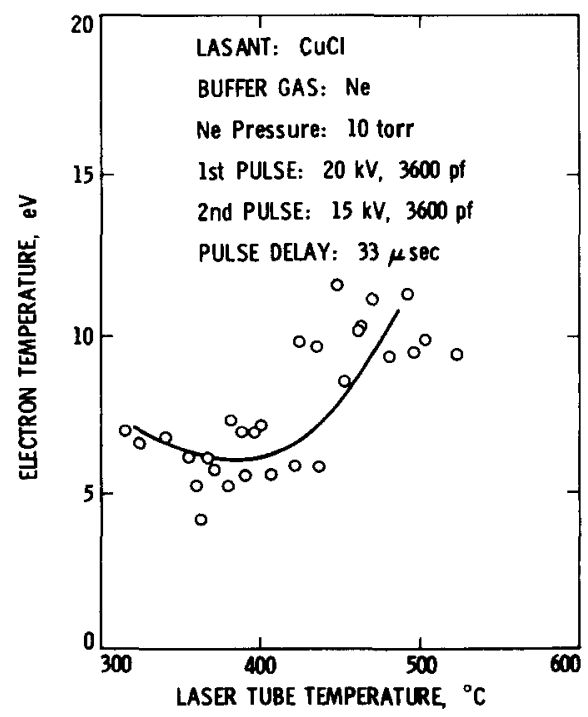

FIG. 8. Electron temperature as function of laser tube temperature for mixture of copper chloride and $\mathrm{Ne}$. 
tron temperature curve agrees in trend with Von Engel's theory, while the ascending portion of the electron temperature curve is contrary to theory. Both curves exhibit a minimum electron temperature at the laser tube temperature of about $400^{\circ} \mathrm{C}$ which is the optimum temperature for maximum laser output for the present experimental arrangement.

\section{B. Possible causes of electron temperature increase}

From Figs. $4-7$ it is apparent that the increase of the electron temperature at higher laser tube temperatures is due to the interaction of buffer gases (He or $\mathrm{Ne}$ ) and the lasant in the tube. The detailed mechanism of the interaction, at this moment, is not known. Possible causes may be Penning ionization and charge exchange ionization of the lasant atoms. The reactions can be represented as follows:

He metastable Penning ionization:

$$
\begin{aligned}
\mathrm{He}\left(2^{1} S, 2^{3} S\right)+X & \rightarrow \mathrm{He}\left({ }^{1} S_{0}\right)+\left(X^{+}\right)^{*}+\mathrm{e} \\
& \rightarrow \mathrm{He}\left({ }^{1} S_{0}\right)+X^{+}+\mathrm{e}+\Delta E_{1} .
\end{aligned}
$$

Charge exchange ionization:

$$
\mathrm{He}^{+}+X \rightarrow\left(X^{+}\right)^{*}+\mathrm{He} \rightarrow X^{+}+\mathrm{He}+\Delta E_{2},
$$

where $X$ can be dissociated $\mathrm{Cu}$ and $\mathrm{Cl}$. In reaction (4), the metastable-state $\mathrm{He}$ atom having excitation energy of about $20.3 \mathrm{eV}$ collides with the copper (chlorine) atom which has an ionization potential of $7.7 \mathrm{eV}(13 \mathrm{eV})$. The collision results in a ground-state He atom, a highly excited copper ion having an excitation energy of $\Delta E_{1}$ $=12.6 \mathrm{eV}(20.3-7.7 \mathrm{eV})$, and a low-energy electron. Similarly for chlorine, the excitation energy $\Delta E_{1}$ is released when it is deexcited. In reaction (5) the He ion exchanges charge with the copper (chlorine) atom, yielding a ground-state He atom and a highly excited $\mathrm{Cu}(\mathrm{Cl})$ ion. The excitation energy in the $\mathrm{Cu}$ ion is $\Delta E_{2}$ $=16.8 \mathrm{eV}$, which is equal to the difference between the ionization potential of $\mathrm{He}(24.5 \mathrm{eV})$ and that of $\mathrm{Cu}(7.7$ $\mathrm{eV})$. For chlorine, $\Delta E_{2}$ is $11.5 \mathrm{eV}$. The $\mathrm{Cu}(\mathrm{Cl})$ ion excitation energy is released through deexcitation. The metastable energy is $4 \mathrm{eV}$ lower with $\mathrm{Ne}$. Evidence of the above reactions has been demonstrated by a previous study ${ }^{8}$ of the $\mathrm{Cu} I I$ laser. The energies $\left(\Delta E_{1}\right.$ and $\left.\Delta E_{2}\right)$ are transferred to the electron gas and radiation by collisional radiative processes. ${ }^{9}$

There is no experimental or theoretical data known to the authors for the rates of the reactions expressed in reactions (4) and (5) for copper or chlorine in the literature. However, the published cross sections for Penning ionization of the metastable state for He with various target species listed in Ref, 10 are higher than $10^{-15} \mathrm{~cm}^{2}$ (with the exception of $\mathrm{H}_{2}$ ). The cross section for Penning ionization of the metastable state for $\mathrm{Ne}$ is about $50 \%$ less than that of $\mathrm{He}$. The charge-exchange cross section can only be inferred from the data for the other elements ${ }^{11}$ to be about $10^{-16} \mathrm{~cm}^{2}$. The numbers quoted here are uncertain and are used only for a qualitative illustration. The reaction characteristic times $\tau_{p}$ and $\tau_{c}$ for the Penning and charge-exchange ionization, respectively, can be estimated by using the quoted numbers with the expression

$$
\tau=1 / q n v \text {, }
$$

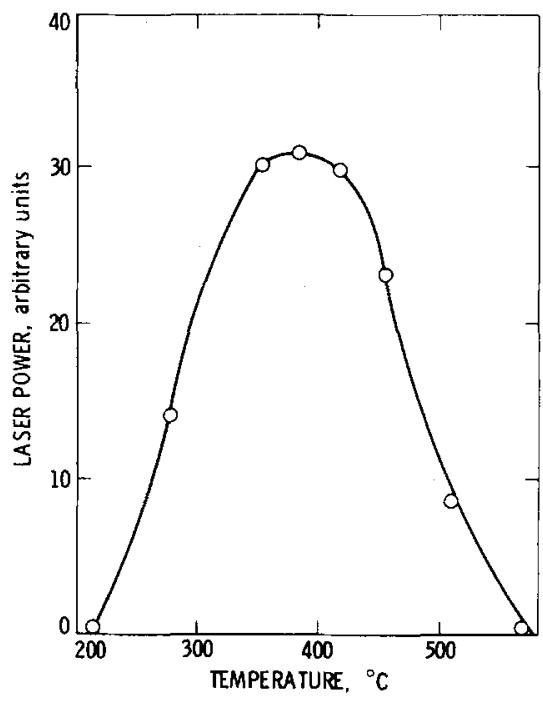

FIG. 9. Typical laser output as function of laser tube temperature.

where $q$ is the ionization cross section $\left(10^{-15} \mathrm{~cm}^{2}\right.$ for $\tau_{p}, 10^{-16}$ for $\left.\tau_{c}\right), n$ is the He metastable state or ion number density $\left(3 \times 10^{16}\right.$ estimated to be $10 \%$ of the He atom density), and $v$ is the thermal speed of the reactants $\left(2 \times 10^{5} \mathrm{~cm} / \mathrm{sec}\right)$.

$$
\begin{aligned}
& \text { Thus, } \\
& \tau_{p} \approx 80 \mathrm{nsec}, \\
& \tau_{c} \approx 1 \mu \mathrm{sec} .
\end{aligned}
$$

$\tau_{p}$ is within the time scale of the present experiment. Thus, Penning ionization may be important in the present experiment.

In comparing Figs. $4-7$ it can be seen that at a tube temperature of about $500^{\circ} \mathrm{C}$ the increases of electron temperature due to the interaction between the copper and the buffer gases are $18 \mathrm{eV}$ for $\mathrm{He}$ and $8 \mathrm{eV}$ for Ne. The magnitude of the increase in electron temperature for a given metastable number density is proportional to the product of the metastable-level energy and Penning-ionization cross section. In the case of $\mathrm{Ne}$, the metastable-level energy $(16.6 \mathrm{eV})$ is smaller than that of He (metastable energy, 20.6 eV), and the Penning-ionization cross sections for $\mathrm{He}$ and $\mathrm{Ne}$ are not expected to be very much different. The measured increases of electron temperature, assumed due to Penning ionization, for the two buffer gases $(\mathrm{He}=18 \mathrm{eV}$, $\mathrm{Ne}=8 \mathrm{eV}$ ) have qualitatively borne out the measurements.

It is also possible that $\mathrm{Cl}_{n}^{-}$negative ions may be formed in the discharge tube causing the electron density to decrease and electron temperature to increase. The result from an independent electron density measurement utilizing the time-resolving Langmuir probe under the same experimental conditions indicate that with the mixture of copper chloride and helium in the discharge tube the time rate of electron density decay $\left(10^{17} \mathrm{sec}^{-1}\right)$ is much slower than that in pure helium $\left(10^{19} \mathrm{sec}^{-1}\right)$. This information supports the postulate of Penning ionization to be the dominant mechanism. 


\section{Explanation of laser output as a function of laser tube temperature}

The typical laser output as a function of laser tube temperature is shown in Fig. 9. From the results discussed in Secs. IV A and IV B, the characteristic of the copper laser represented by the curve in Fig. 9 can be explained as follows. At lower laser tube temperature (in the vicinity of $300^{\circ} \mathrm{C}$ ) the copper number density is low $\left(5.5 \times 10^{12} \mathrm{~cm}^{-3}\right)$. The lack of copper-atom number density explains the lower laser output in this range of laser tube temperatures. At higher laser tube temperatures (in the vicinity of $500^{\circ} \mathrm{C}$ ) the number density of copper is $6 \times 10^{15}$ following the dissociation of copper chloride. For the elevated electron temperatures (with $\mathrm{He}, 25 \mathrm{eV}$ and with $\mathrm{Ne}, 13 \mathrm{eV}$ ) the copper should be fully ionized, again producing a lack of copper atoms. To support this argument, the ionization characteristic times for ionization of copper atoms are estimated. The experimental value of the ionization rate of copper atoms by electron collisions is not available. However, the ionization rate by electron collision as a function of electron temperature is calculated by using the Gryzinski classical formulation ${ }^{12}$ with a Maxwellian electron velocity distribution. The electron temperatures are 25 and 13 $\mathrm{eV}$ for He and Ne buffer gases, respectively. The characteristic times for ionization of copper atoms in $\mathrm{He}$ and Ne buffer gases are estimated to be $10^{-8}$ and $3 \times 10^{-8}$ $\mathrm{sec}$, respectively, which are in the range of characteristic times for the experiments.

The optimum laser power is obtained in the laser tube at a temperature of about $400^{\circ} \mathrm{C}$. The corresponding electron temperatures, as shown in Figs. 4 and 5 , are about $6-7 \mathrm{eV}$. At these electron energies the ionization rate (ionization characteristic time is about $10^{-6} \mathrm{sec}$ ) is not high enough to deplete the copper-atom number density and cause deterioration of the laser output.

\section{ACKNOWLEDGMENT}

The authors are indebted to Dr. Raymond Goldstein for the calculation of copper-atom ionization rates and to James Yocom for his technical assistance.

*Work sponsored by the Defense Adranced Research Projecls Agency (DARPA) and the Caltech President's Fund.

${ }^{1}$ C.J. Chen, N.M. Nerheim, and G. R. Russell, Appl. Phys. Lett. 23, 514 (1973).

${ }^{2}$ G. Bekefi and S. C. Brown, J. Appl. Phys. 32, 25 (1961).

${ }^{3} \mathrm{M}$. Sugawara and B. C. Gregory, Phys. Rev. 2, A439 (1970).

${ }^{4} \mathrm{G}$. Bekefi, Radiation Processes in Plasma (Wiley, New York, 1966), p. 55.

"G. Bekefi, J.L. Hirshfield, and S. C. Brown (unpublished).

${ }^{6}$ Lymen Spitzer, Jr., Physics of Fully Ionized Gases (Interscience, New York, 1956).

'A. Von Engel and M. Steenbeck, Electrische Gasentladungen, ihre physik and technik (Springer-Verlag, Berlin, 19:32), Vol. 2 , p. 86 .

${ }^{8} \mathrm{~J} . \mathrm{R}$. McNeil and G.J. Collins, Appl. Phys. Lett. 27, 595 (1975).

${ }^{9} \mathrm{Che}$ Jen Chen, Phys. Rev. 163, 1 (1967).

${ }^{10}$ W. P. West, T.B. Cook, F.B. Dunning, R. D. Rundel, and R. F. Stebbings, J. Chem. Phys. 63, 1237 (1975).

${ }^{11}$ S. C. Brown, Basic Data of Plasma Physics (Wiley, New York, 1959), pp. 36-45.

${ }^{12}$ M. Gryzinski, Phys. Rev. 138, A336 (1965). 